\title{
The correlation between the mean voltage recorded during atrial fibrillation and sinus rhythm during substrate mapping of the atria
}

\author{
Bita Behrouzi ${ }^{1}$, Kathryn Hong ${ }^{2}$, Maria Terricabras ${ }^{3}$, Adam Korogyi ${ }^{4}$, Jason Baley ${ }^{5}$, Benci \\ Galapin $^{2}$, Atul Verma ${ }^{6}$, and Benedict Glover ${ }^{7}$ \\ ${ }^{1}$ University of Toronto \\ ${ }^{2}$ Schulich Heart Center \\ ${ }^{3}$ Hospital Universitari Vall d'Hebron \\ ${ }^{4}$ Abbott technologies \\ ${ }^{5}$ Abbott \\ ${ }^{6}$ Southlake Regional Health Centre \\ ${ }^{7}$ Toronto General Hospital
}

July 6, 2020

\begin{abstract}
There is currently no correlation between substrate mapping during atrial fibrillation and sinus rhythm. We sought to determine the correlation between the mean voltage recorded during atrial fibrillation and sinus rhythm at different times up to a total duration of 30 seconds. We found that the most accurate correlation between the mean voltage occurred over a period of 10 seconds recording. Although in average the mean voltage during atrial fibrillation is lower than during sinus rhythm this represents the most accurate comparison between voltage mapping during atrial fibrillation and sinus rhythm.
\end{abstract}

\section{Introduction}

Atrial fibrosis is the most important predictor of arrhythmia recurrence following pulmonary vein (PV) isolation and is a marker of AF persistence and progression. [1] Despite the importance of this there is no common consensus on the optimal method of visualizing fibrosis. Delayed enhanced magnetic resonance imaging often lacks adequate resolution and has a significant potential for artefact. [2] Although high density bipolar voltage mapping during sinus rhythm (SR) may be considered a surrogate for the presence of fibrosis [3] there are many potential factors which may influence these recordings including wavefront dynamics, electrode orientation, spacing and size. [4] Importantly many patient who attend for catheter ablation for $\mathrm{AF}$ are not in SR at the time of the procedure and there is no clear correlation between substrate mapping during either arrhythmia. Voltage recordings during $\mathrm{AF}(\mathrm{AFv})$ are calculated as the maximum peak to peak within a window of interest and vary significantly from voltage recordings made during sinus rhythm (SRv).

The objective of our study was to assess whether there is a clear relationship between the mean AFv recorded over various durations and the SRv.

\section{Methods and Results}

This study was approved by the Institutional Review Board and data was obtained from nine consecutive patients with drug-refractory AF undergoing a first time catheter ablation. Bipolar electrograms of the left atrium (LA) were recorded using a 24 pole flat array catheter (HD Grid, Abbott technologies, Mn, USA) prior to the ablation procedure. A total of 7 specific anatomical locations were mapped in SR and during AF: the anterior LA appendage, the ostium of each of the four PV's, the posterior wall and the lower septum. 
A shadow of the catheter location was created to ensure standardization of the location during both SR and AF. During AF mean peak-to-peak bipolar voltage amplitude were computed in time periods of $1 \mathrm{~s}, 3 \mathrm{~s}, 10 \mathrm{~s}$, 20s, and 30s for AF and compared with a single SR beat.

The comparison of continuous variables was assessed with ANOVA test. Correlation between the mean amplitude recorded during AF with SR was assessed with Spearman correlation coefficients, and robust linear regression was performed to reduce outlier effect. A p-valve of less than 0.05 was considered statistically significant. All analysis were performed using Matlab R2018b.

A total of 4352 electrical points were recorded, 2104 during sinus rhythm and 2248 during AF from 16 patients (mean age $64+/-8$ years, $5 / 9$ female; median duration of AF 7 (3-10) months). As shown in Figure 1 the mean AFv was significantly lower compared to $\mathrm{SRv}(0.5536 \pm 0.7767 \mathrm{mV}$ vs $1.1373 \pm 2.0497, \mathrm{p}<$ 0.001). There was a moderate to strong correlation between $\mathrm{SRv}$ and $\mathrm{AFv}$ for all the different durations of voltage acquisition: 0.698 for $1 \mathrm{~s}, 0.601$ for $3 \mathrm{~s}, 0.892$ for $10 \mathrm{~s}, 0.715$ for $20 \mathrm{~s}$, and 0.70963 for $30 \mathrm{~s}$. There was an excellent correlation between the 10 second duration recording $(r=0.892 ; \mathrm{p}<0.0001)$ and the Srv. This relationship was seen throughout all regions of the LA.

In our study the mean voltages recorded throughout the LA were lower during AF than SR which is in keeping with a recent study in which the voltage recorded during AF demonstrated a better correlation than SR when compared with a DE-MRI of the posterior wall of the LA. [5] This study also demonstrated that $\mathrm{AF}$ voltage recordings tend to stabilize after a period of 4 seconds. For the first time we have found a linear correlation between the voltage amplitude in SR and AF with adequate temporal sampling in the most common locations for substrate. The disorganized wavefront activation during AF results in variability in every peak to peak voltage amplitude but mapping during a longer period of time showed a good correlation with the electrograms in SR.

\section{Conclusion}

A recording of 10 seconds provided an excellent correlation with voltages recorded during SR. Interestingly there was no additional benefit when sampling beyond this. This should be considered to be incorporated into an algorithm for substrate mapping during AF in the future.

\section{References}

1. Verma A, Wazni OM, Marrouche N.F. et al. Pre-existent left atrial scarring in patients undergoing pulmonary vein antrum isolation: an independent predictor of procedural failure. J Am Coll Cardiol 2005: 45:285-292

2. Benito, E.M., Carlosena-Remirez, A., Guasch, E. et al. Left atrial fibrosis quantification by late gadolinium-enhanced magnetic resonance: a new method to standardize the thresholds for reproducibility. Europace. 2017: 19: 1272-1279

3. Oakes RS, Badger TJ, Kholmovski EG, et al. Detection and Quantification of Left Atrial Structural Remodeling With Delayed-Enhancement Magnetic Resonance Imaging in Patients With Atrial Fibrillation Circulation 2009: 119:1758-67

4. Josephson M.E., Anter E. Substrate mapping for ventricular tachycardia. JACC Clin Electrophysiol. $2015 ; 1: 341-352$

5. Qureshi NA, Kim SJ, Cantwell CD, et al. Voltage during atrial fibrillation is superior to voltage during sinus rhythm in localizing areas of delayed enhancement on magnetic resonance imaging: An assessment of the posterior left atrium in patients with persistent atrial fibrillation. Heart Rhythm. 2019 Sep; 16: $1357-1367$

Figures

Figure 1: Voltage map of the left atrium (posterior view) recorded during sinus rhythm on the left and atrial fibrillation on the right using the Advisor HD Grid Mapping Catheter, Sensor Enabled ("HD Grid", Abbott, St. Paul, MN, USA) catheter. Regions of lower voltage are seen in the posterior wall during 
atrial fibrillation which is not evident during sinus rhythm. This corresponds to lower amplitude waveforms recorded and shown at the bottom of the image during atrial fibrillation when compared to sinus rhythm.

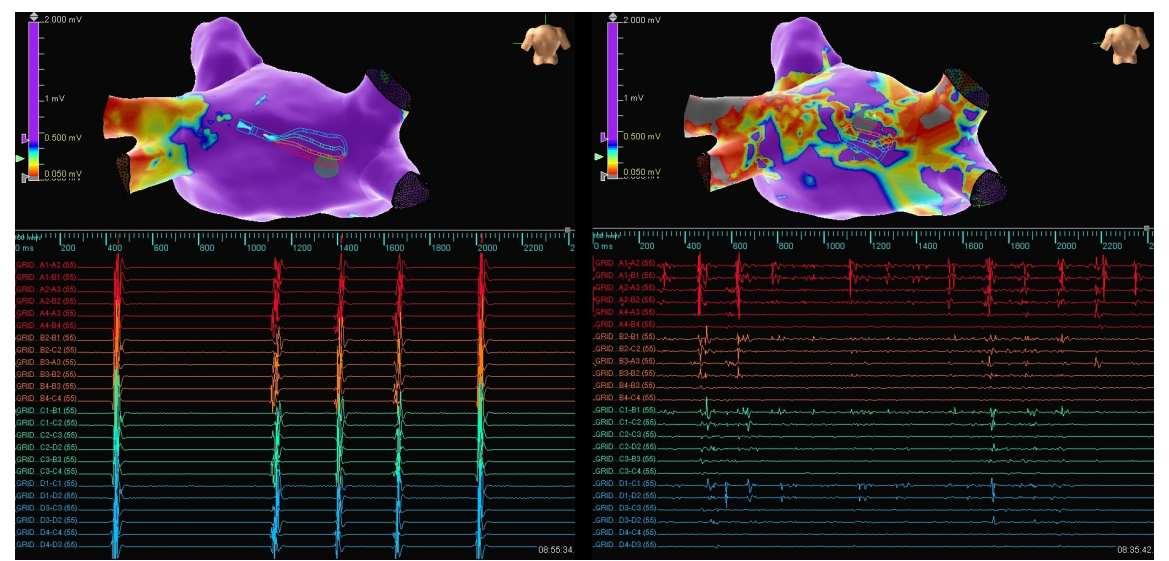

